\title{
La norma en lo cotidiano del mobiliario de la Caja del Seguro Obrero Obligatorio(1)
}

\section{Resumen}

El presente artículo intenta exponer cómo el diseño participa de una configuración de la realidad desde una dimensión político administrativa, siendo incluido desde una perspectiva moderna, expresada en la construcción de una imagen normada de lo cotidiano.

El caso de estudio presentado es el de la Caja del Seguro Obrero Obligatorio, o la historia de cómo a partir de la concepción de una institucionalidad vinculada al Estado asistencialista de la primera mitad del siglo XX, y de su correspondiente estructura administrativa, se construye una nueva realidad, sin precedente, donde la protección de la vida y el despliegue de los instrumentos requeridos convocan un correlato en arquitectura, vestuario y diseño de mobiliario, una realidad "otra" que se desarrolla o se desenvuelve y dispersa en el territorio, a partir de una concepción abstracta inicial, y que repercute en expresiones de diseño de extraordinaria calidad y personalidad.

La Caja del Seguro Obrero Obligatorio fue una institución creada en 1924 por la ley $4.054^{(2)}$ que dio origen al primer sistema asistencial estatal de salud y de previsión para la vejez y accidentes del trabajo; su vida productiva se extendió en propiedad desde 1935 hasta $1952^{(3)}$, coincidiendo en gran parte con la consolidación de una modernización del Estado chileno, la cual tuvo un desarrollo relevante con las acciones del gobierno de Carlos Ibáñez del Campo a partir de $1927^{(4)}$.

Esta voluntad modernizadora, cuyas bases descansan en el proyecto de la ilustración, que otorgaba a la razón el privilegio del orden comprendía, devenido de la misma base, un ideal de progreso social que fue canalizado por el Estado a través de la modificación, fusión y 
creación de nuevas instituciones encargadas de beneficiar a aquellos segmentos más desposeídos de la sociedad de fines del siglo XIX y principios del siglo XX.

En Chile, las políticas sociales de los gobiernos de las primeras décadas del siglo XX fomentaron una serie de iniciativas para construir una infraestructura acorde al sentido social del Estado benefactor en las áreas de vivienda, educación, salud y administración pública. Santiago, en su condición de ciudad capital y de mayor concentración de población del país, fue equipada de un importante número de edificios proyectados y construidos para servir a esos fines.

En este sentido, en la disposición de servicios se desplegaba la capacidad ordenadora como facultad del Estado moderno que homogeneizaba y regulaba el comportamiento de todos sus individuos y cualquier otra organización en sus fronteras ${ }^{(5)}$.

La ley 4.054, según el médico y abogado Hermes Ahumada, "Fue la primera ley de este carácter en América Latina y en nuestro país que inició una legislación de Seguridad Social que significó, más que

Imagen 1 . una tentativa legislativa, una ordenación de una política de seguridad Edificio de la Caja del Se- social”"(6).

guro Obrero Obligatorio. Esta política de ordenación se materializó mediante la conformaArquitecto Ricardo Gon- ción de la Caja del Seguro Obligatorio que constituyó una zález Cortés, 1928. organización de gran envergadura que mediante fondos provenientes Fuente: Autor. principalmente del Estado, de los afiliados y los empleadores extendió

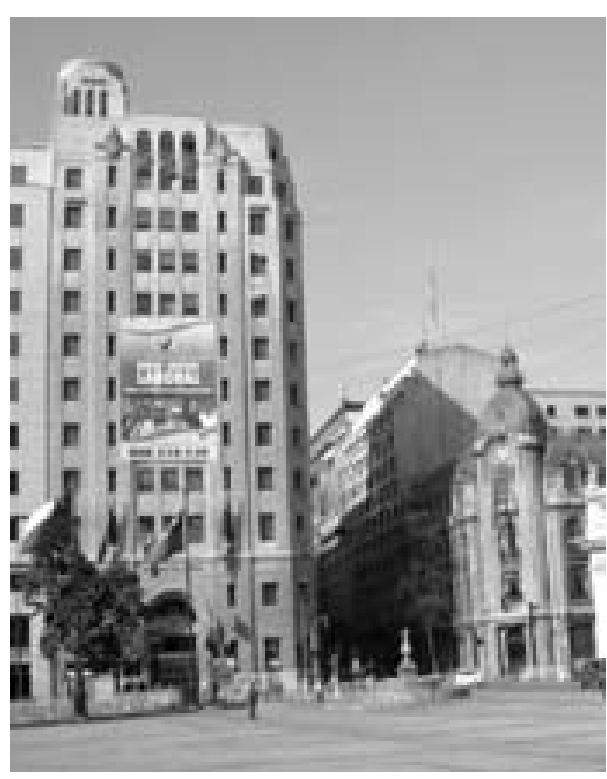
una relevante red de servicios médicos y previsionales no solo referente a la ciudad de Santiago, sino a nivel nacional ${ }^{(7)}$.

Por otra parte, la ley 4.054 determinaba que la Caja Central y sus filiales podrían establecer dispensarios, farmacias, hospitales, sanatorios, casas de convalecencia y laboratorios de diagnóstico y estableció una organización en base a una Caja Central y Cajas Locales.

Para llevar a cabo sus objetivos a lo largo del país, la Caja del Seguro Obrero se estableció en Santiago y en el año 1928 fue construido el edificio para el funcionamiento de las dependencias centrales por el arquitecto Ricardo González Cortés, siendo actualmente el inmueble que utiliza para sus funciones el Ministerio del Trabajo.
Progresivamente, en regiones fue consolidado el servicio de la Caja del Seguro Obrero. En i93 I se creó la Caja de Viña del Mar y en 1934 la Caja Local de Lota. Asimismo las ciudades de Concepción, Valparaíso, Coquimbo, entre otras, contaron con Cajas Locales pertenecientes a la Caja del Seguro Obrero. Las prestaciones médicas se llevaron a cabo mediante inmuebles construidos o adaptados que la Caja levantó o adquirió a lo largo del país. En Santiago, los edificios más destacados fuero el Consultorio $\mathrm{N}^{\circ}$ I y el Consultorio $\mathrm{N}^{\circ}$, entre más de treinta inmuebles destinados en distintas escalas a la administración de salud en la ciudad de Santiago.

La Caja cubría asimismo otras necesidades de los afiliados como por ejemplo, asociadas a las prestaciones médicas, la distribución de medicamentos y leche ${ }^{(8)}$; la adquisición y arriendo de viviendas, y en 1933 inicia la Caja de Servicio de Vestuario.

En 1937 con la incorporación de la Sección Médico Social a la Caja, destinada a organizar la labor médico preventiva ${ }^{(9)}$, el Servicio Social, existente desde los inicios de la institución, sección Educación Sanitaria, existente desde $1937^{(10)}$, y Sección Dental, que acompañó los inicios de la Caja, el servicio sostenía un cercano vínculo con sus más de 80.000 afiliados.

Esta amplia cobertura del servicio de salud, previsión, habitacional y de orden tan rutinarias como el vestuario, normaba y regulaba, por parte del Estado, tanto la administración de los mismos, que anteriormente se encontraban en manos de cajas particulares y de la beneficencia; como las prácticas médicas entregadas a la población.

Por otra parte, normalizaba y periodizaba, ingresando en el discurso de lo cotidiano, la vinculación de los afiliados con la institución configurando una realidad homogeneizada, en lo referente al suministro de salubridad, tanto por las prácticas médicas así como por las rutinas de los afiliados asociadas a estas prácticas.
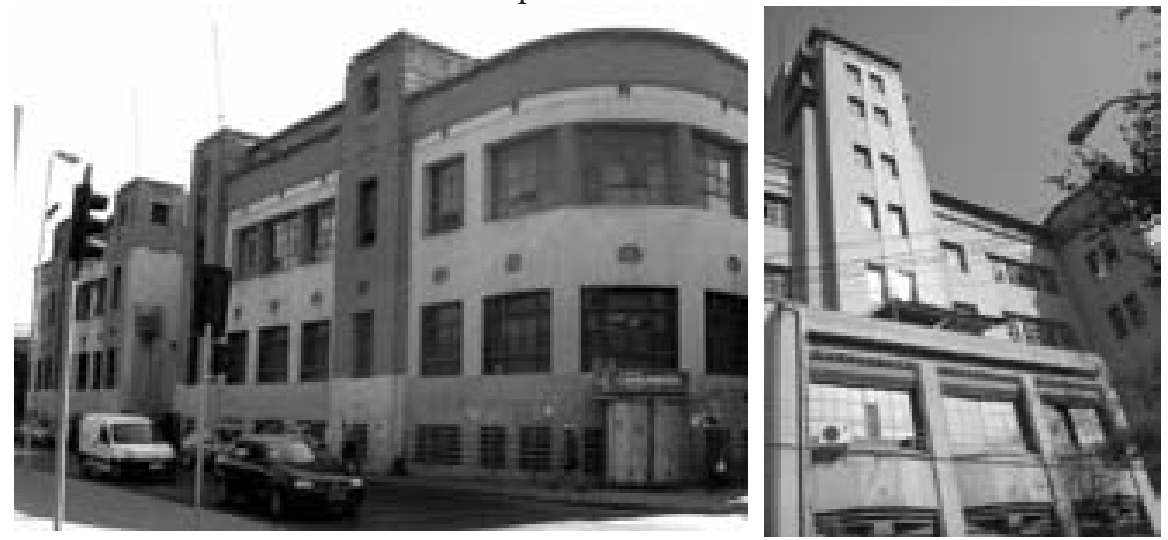
Asimismo, la organización de las actividades al interior de los consultorios y centros de tratamiento se encontraba definida, lo que se reflejó en la destinación a ciertas actividades fijas en cada inmueble, quedando incluso el mobiliario estandarizado para el desarrollo de éstas.

En 1935 la Caja del Seguro Obligatorio publica las "Especificaciones de muebles para los Policlínicos y Cajas Locales del Seguro Obligatorio, tipo standard"(Ir), en las que consigna un grupo de doce Imagen 3. muebles: a) estante-archivador, para oficinas administrativas, poliEstante-archivador, para clínicos y postas rurales; b) mesa escritorio para jefes de cajas locales, oficinas administrativas, policlínicos departamentales, calculadores de subsidio y empleados policlínicos y postas rurales. en general; c) mesa escritorio para médicos tratantes, postas rurales, Fuente:Especificaciones de casas de socorro y practicante; d) escritorio alto para llevar libros o muebles para los policlínicos y atención de público; e) estante para hojas clínicas; f) estante para cajas locales del seguro obliga- las libretas; g) mesa escritorio para administradores y médicos ditorio, tipo Standard. Santiago rectores provinciales; h) mesas para máquina de escribir; i) mesón 1934. Impr. Lathrop Hnos. de atención del público de dimensiones variables de acuerdo a los
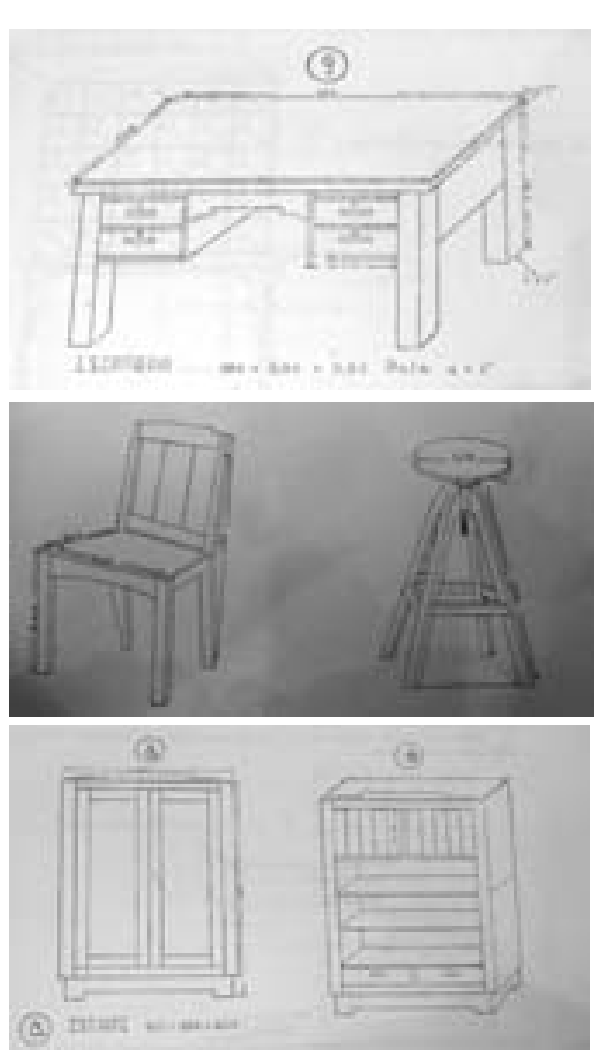

ocales. Sillas tapizadas en cuero o pegamoide para todas las oficinas, policlínicos y casas de socorro. Pisos altos para oficinas y policlínicos.

En lo referente a materialidad, se consignaba el uso de maderas de raulí o lingue de primera clase con barniz exterior a muñequilla aclarado, interior a la cera, color nogal; siendo el tapiz en cuero sin resortes, los pisos, giratorios. En cuanto a la factura, se estipulaba que todos los trabajos se ejecutarían en diajustes, ensamblados y cubiertas atarugadas, cajones endentados y cerraduras de garganta. Las dimensiones de las maderas se precisaban en los planos, siendo todo esto de primera calidad ${ }^{(12)}$.

Esta homogeneización de la realidad, que configuraba una imagen de los servicios estatales brindados a la población elaborada desde una racionalización del sistema político-administrativo, se expresó en la aplicación de un lenguaje arquitectónico externo y de diseño interior, común con los objetos de diseño de uso cotidiano como el mobiliario (se podría decir que incluso en vestuario) compartiendo un sentido estéti- co de simplificación formal que privilegiaba la funcionalidad por sobre la aplicación de elementos ornamentales.

Si bien, desde la perspectiva de la arquitectura, la inserción de las tendencias estilísticas pre-racionalistas como el "Art Deco", presente en los edificios aquí mencionados ${ }^{\left({ }^{13}\right)}$, ha sido poco investigada y sistematizada, es posible observar en numerosas edificaciones de viviendas e inmuebles públicos elementos coincidentes de estas características en los años 30, como antecedentes y previos al desarrollo de la imagen de un racionalismo maduro. Sin duda esta expresión constituye un distanciamiento del lenguaje imperante proveniente del repertorio formal instalado mediante el "Beaux Arts", vigente aun en el periodo en la enseñanza de las escuelas de arquitectura del país.

En el campo del arte, sin embargo, la discusión había tomado otros rumbos desde la década del 20 , específicamente con la reforma a la enseñanza artística impulsada en el gobierno de Carlos Ibáñez del Campo en 1927 -al alero de la modernización del aparataje estataly la reorganización del plan de estudio de la Academia de Bellas Artes dirigida por Carlos Isamit ${ }^{(14)}$, que privilegió la creación de una escuela de Artes Aplicadas al interior de la Academia en vista a potenciar un nuevo sentido estético aplicable a la producción industrial superando los preceptos academicistas ${ }^{(15)}$ con un carácter racionalista y nacionalista.

Tanto en el caso de la arquitectura como en el del mobiliario, este sentido de simplificación formal acompañaba un criterio moderno en tanto económico de racionalización de recursos y específicamente en el caso de los muebles aquí tratados, una capacidad productiva y técnica que acusaba su edad, proveniente de un estado aún más vinculado a la fabricación artesanal que a la industrial.

La unidad de la imagen en la escala de esta primera red de servicios estatales, desde la arquitectura hasta el diseño de interiores y de mobiliario, configuró por cerca de dos décadas un escenario de cotidianidad racionalizada, normada en tanto asociada a todos los procedimientos periodizados de administración de salud estatal para un gran segmento de la sociedad chilena que utilizaba estos recursos.

En 1952, mediante la ley 10.383 la Caja del Seguro Obrero fue fusionada con otras instituciones de similares objetivos para formar el Ministerio de Salud y el Servicio de Seguro Social, sus inmuebles y muebles pasaron a formar parte de estas unidades en lo referente a las prestaciones de salud y a las pensiones de invalidez y vejez respectivamente fragmentando una dimensión conceptual de racionalidad en tanto protección de la vida, expresada en la construcción de una imagen normada de lo cotidiano. 
1. El presente artículo se desprende de la información anexa recopilada por la autora, para el proyecto de investigación "Red de Consultorios de la Caja del Seguro Obrero. Un patrimonio emblemático", adjudicado mediante fondos de la Vicerrectoría de Investigación y Desarrollo de la Universidad de Chile, en el periodo 2011-2012. Investigador responsable: Alicia Campos G. Coinvestigadores Patricio Basáez Y, Marcela Pizzi K. María Paz Valenzuela B. Facultad de Arquitectura y Urbanismo. Universidad de Chile.

2. Ley 4.054 del 8 de Septiembre de 1924.

3. De acuerdo a lo observado por Biondi A y otros. Caja del Seguro Obligatorio. 1924-1944. Desarrollo estructural y funcional. Racionalización de los servicios y prestaciones. "Boletín Médico Social. Caja del Seguro Obligatorio". Año XI Stgo. Julio- septiembre 1944

4. Mediante la ley $N^{\circ} 4.113$ con la que se creaban nuevos organigramas y plantas de los servicios con objeto de optimizar recursos y el número de empleados fiscales, en conjunto con la ley 4.156, que complementaba a la primera, autorizaba la reorganización de los ministerios mediante decreto presidencial. Redefinió el número, el nombre y las funciones de los Ministerios de Interior, Relaciones Exteriores, Hacienda, Educación Pública, Justicia, Guerra y Marina, Fomento (que comprendía los de Agricultura y Obras Públicas) y Bienestar Social, antes Higiene, Asistencia, Previsión Social y Trabajo.

5. Atendiendo a la perspectiva de Estado planteada por Ted Honderich. "State" In Honderich Ted. (ed). The Oxford Companion on Philosophy. Oxford New York. Oxford University Press. 1995. Pág. 580.,

6. Ahumada Hermes. Manual de Seguridad Social. Santiago, Ed. Andrés Bello.

7. Los beneficios para los afiliados fueron los siguientes: Asistencia médica y provisión de todos los medios terapéuticos necesarios. Hospitalización del enfermo, en caso de ser ordenado por el médico. Personal a disposición para la atención del asegurado, dispensada mediante la Caja Subsidio en dinero mientras duraba la incapacidad del asegurado, con objeto de cubrir los gastos familiares. Atención profesional para las aseguradas embarazadas, más un $50 \%$ de salario durante las tres primeras semanas después del nacimiento y un $25 \%$ durante el periodo posterior. Una suma en pesos a entregar a la familia en caso de fallecimiento del asegurado. Pensión de invalidez en caso de accidentes que produjeran la incapacidad de trabajar para el asegurado. Dicha pensión fue determinada igual al salario promedio obtenido por el asegurado en el último año y variaba de acuerdo a la antigüedad de la pertenencia a la Caja. Pensión de Invalidez de carácter etaria (sobre los 65 años, superando los 15 años de afiliación a la Caja).

8. Para lo cual adquirió el Laboratorio Chile y construyó la Central de Leche.

9. Específicamente en lo referente a exámenes de tuberculosis y enfermedades venéreas.

10. Que tenía como objetivo impartir conocimientos prácticos sobre higiene general mediante diversos soportes gráficos.

11. Santiago: Impr. Lathrop Hnos. 1934.

12. Caja del Seguro. Op Cit. Pág. 2

13. Detalles ornamentales del edificio de la Caja del Seguro Obrero de Ricardo González Cortés y Consultorios $N^{\circ} 1$ y $N^{\circ} 2$ diseñados por la oficina de arquitectura de la
Caja del Seguro Obligatorio.

14. Al respecto, ver Lizama, P. "El cierre de la Escuela de Bellas Artes en 1929. Propuestas, querellas y paradojas de la Vanguardia Chilena". En Revista Ahistesis $\mathrm{N}^{\circ} 34$.

15. Cuyo fracaso devino en la inclusión de la Academia a la organización de la Universidad de Chile.

\section{Bibliografía consultada}

Ahumada, H. (1972). Manual de Seguridad Social. Santiago, Chile: Ed. Andrés Bello.

Cruz-Coke, E. (1993). Historia de la medicina chilena. Santiago, Chile: Ediciones de la Universidad de Chile.

Foucault, M. (2007). Nacimiento de la Biopolitica: Curso en el College de France (1978-1979). Buenos Aires, Argentina: Fondo de Cultura Económica.

Illanes, M.A. (1993). En el nombre del pueblo, del estado y de la ciencia: historia social de la salud pública en Chile 1880/1973. Santiago, Chile: Colectivo de Atención Primaria.

Lizama, P. (200I). El cierre de la Escuela de Bellas Artes en 1929. Propuestas, querellas y paradojas de la Vanguardia Chilena. Revista Ahistesis, (34).

Mardones, J. (1954). La reforma de la seguridad social de los obreros: motivos de la Ley 10.383 Restat. Santiago, Chile: Impr. Enc. Hispano-Suiza.

Vargas, N. (2002). Historia de la Pediatría Chilena. Santiago, Chile: Ed. Universitaria.

Vial, G. (1994). Historia de Chile. Santiago, Chile: Ed. Santillana del Pacífico.

Caja del Seguro Obligatorio. Boletín Médico Social [boletín]. (1944 julio-septiembre). Santiago, Chile.

Ley n ${ }^{\circ} 10.383$ del Seguro Social Obligatorio. Diario Oficial, Santiago, Chile, 8 de agosto de 1952.

Ley 4.054 del Seguro Obrero Obligatorio de Enfermedad e Invalidez. Ediciones de la Empresa Periodística. Santiago. 1925 .

Órgano Oficial del Centro de Investigaciones de Historia de la Medicina de la Universidad de Chile y de la Sociedad Chilena de Historia de la Medicina. Anales chilenos de historia de la medicina. 